\title{
DOA ESTIMATION EXPLOITING COPRIME ARRAYS WITH SPARSE SENSOR SPACING
}

\author{
Yimin D. Zhang, Si Qin, and Moeness G. Amin \\ Center for Advanced Communications, Villanova University, Villanova, PA 19085, USA
}

\begin{abstract}
In this paper, we propose effective coprime array configurations in which the minimum interelement spacing is much larger than the typical half-wavelength requirement. Such configurations are important in many applications where the half-wavelength requirement cannot be met due to the physical sensors size or to avoid spatial oversampling in wideband operations. The application of such coprime arrays in direction-of-arrival estimations is examined using different algorithms.
\end{abstract}

Index Terms - Coprime array, sparse array, difference co-array, direction-of-arrival estimation, compressive sensing

\section{INTRODUCTION}

Among a number of techniques that are available for sparse array construction, coprime array is very attractive due to its simplicity and effectiveness [1]. A prototype coprime array utilizes a coprime pair of uniform linear subarrays, where one is of $\tilde{M}$ sensors with an interelement spacing of $\tilde{N}$ units, whereas the other is of $\tilde{N}$ elements with an interelement spacing of $\tilde{M}$ units. The numbers $\tilde{M}$ and $\tilde{N}$ are chosen to be coprime. The unit interelement spacing, $d$, is typically set as half wavelength, or $\lambda / 2$. Because the two subarrays share the first sensor at the zeroth position, the corresponding coprime array has a total number of $\tilde{M}+\tilde{N}-1$ sensors and achieves $O(\tilde{M} \tilde{N})$ degrees-of-freedom (DOFs), which is much larger than the number of physical array elements. The increased DOFs enable direction-of-arrival (DOA) estimation of more sources than sensors [2]-[6].

While coprime array provides a sparse configuration for array placement, the minimum spacing between the sensors is the unit spacing, which is typically half wavelength to avoid the grating problem. Many other sparse array structures, such as the minimum redundancy array [7], generally share the same property. There are situations, however, that such halfwavelength minimum spacing is not applicable. One of the examples is when the physical size of the antenna sensors is larger than half-wavelength (e.g., [8]). Indeed, many parabola antennas are designed to have a large size for enhanced directivity [9]. Another example is an array that operates over a wide spectrum. For example, over-the-horizon radar (OTHR) is a unique radar system that performs wide-area surveillance by exploiting the reflective and refractive nature of high-frequency $(\mathrm{HF})$ radiowave propagation through the

This work was supported in part by the Office of Naval Research (ONR) under grant N00014-13-1-0061. ionosphere [10]. While OTHR systems employ narrowband signals, typically with a signal bandwidth of tens of kilohertz, the carrier frequency may be chosen to be any value in the HF band (e.g., the U.S. Navy Relocatable Over-the-Horizon Radar (ROTHR) operates between $5 \mathrm{MHz}$ and $30 \mathrm{MHz}$ ). This choice is governed by the ionospheric conditions and the target ranges of interest. As such, the electrical length of a physical array varies significantly with the frequency. Thus, sparse array designs are desirable to avoid undersampling at the high end of the HF band and oversampling at its low end and achieve effective signal radiation and clutter suppression.

In this paper, we build on the coprime concept to design unique array structures that maintain a minimum interelement spacing far above the half-wavelength unit spacing, yet allow adaptive beamforming and direction finding without the grating/aliasing problem. In particular, the two subarrays in the proposed coprime array structure are allocated with a displacement rather than collocated. Such coarray structure is referred to as coprime array with displaced subarrays (CADiS).

The application of such coprime array structure in DOA estimations is examined using MUSIC and compressive sensing (CS) techniques. Compared to the conventional coprime array structures, the CADiS configuration achieves higher number of virtual sensors and DOFs for CS-based DOA estimation, but the consecutive virtual sensors required for MUSIC-based approach is approximately halved.

Notations: We use lower-case (upper-case) bold characters to denote vectors (matrices). In particular, $\mathbf{I}_{N}$ denotes the $N \times N$ identity matrix. (.)* implies complex conjugation, whereas $(.)^{T}$ and $(.)^{H}$ respectively denote the transpose and conjugate transpose of a matrix or vector. $\operatorname{vec}(\cdot)$ denotes the vectorization operator that turns a matrix into a vector by stacking all columns on top of the another, and $\operatorname{diag}(\mathbf{x})$ denotes a diagonal matrix that uses the elements of $\mathbf{x}$ as its diagonal elements. $\|\cdot\|_{2}$ and $\|\cdot\|_{1}$ respectively denote the Euclidean $\left(l_{2}\right)$ and $l_{1}$ norms, and $\mathrm{E}(\cdot)$ is the statistical expectation operator. $\otimes$ denotes the Kronecker product, and real $(\cdot)$ and $\operatorname{imag}(\cdot)$ represent the real and imaginary part operations.

\section{COPRIME ARRAY CONCEPT}

A prototype coprime array, as described in the previous section, is illustrated in Fig. 1. Without loss of generality, we assume $\tilde{M}<\tilde{N}$. The unit interelement spacing $d$ is set to $\lambda / 2$. The array sensors are positioned at

$$
S=\{n \tilde{M} d, 0 \leq n \leq \tilde{N}-1\} \cup\{m \tilde{N} d, 0 \leq m \leq \tilde{M}-1\}
$$




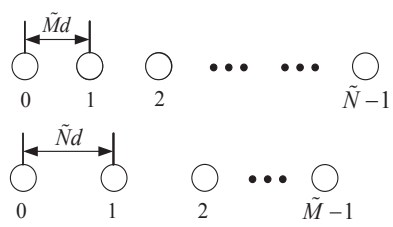

Fig. 1. Configuration of the prototype coprime array.

Because the two subarrays share the first sensor at the zeroth position, the total number of the sensors used in the coprime array is $\tilde{M}+\tilde{N}-1$. Note that the minimum interelement spacing in this coprime array is $\lambda / 2$.

Denote $\mathbf{p}=\left[p_{1}, \ldots, p_{\tilde{M}+\tilde{N}-1}\right]^{T}$ as the positions of the array sensors where $p_{i} \in S, i=1, \ldots, \tilde{M}+\tilde{N}-1$, where the first sensor is assumed as the reference, i.e., $p_{1}=0$. Assume that $Q$ uncorrelated signals imping on the array from angles $\Theta=\left[\theta_{1}, \ldots, \theta_{Q}\right]^{T}$, and their discritized baseband waveforms are expressed as $s_{q}(t), t=1, \ldots, T$, for $q=1, \ldots, Q$. Then, the data vector received at the coprime array is expressed as

$$
\mathbf{x}(t)=\sum_{q=1}^{Q} \mathbf{a}\left(\theta_{q}\right) s_{q}(t)+\mathbf{n}(t)=\mathbf{A} \mathbf{s}(t)+\mathbf{n}(t),
$$

where

$$
\mathbf{a}\left(\theta_{q}\right)=\left[1, e^{j \frac{2 \pi p_{2}}{\lambda} \sin \left(\theta_{q}\right)}, \ldots, e^{j \frac{2 \pi p \tilde{M}+\tilde{N}-1}{\lambda} \sin \left(\theta_{q}\right)}\right]^{T}
$$

is the steering vector of the array corresponding to $\theta_{q}, \mathbf{A}=$ $\left[\mathbf{a}\left(\theta_{1}\right), \ldots, \mathbf{a}\left(\theta_{Q}\right)\right]$, and $\mathbf{s}(t)=\left[s_{1}(t), \ldots, s_{Q}(t)\right]^{T}$. The elements of the noise vector $\mathbf{n}(t)$ are assumed to be independent and identically distributed (i.i.d.) random variables following the complex Gaussian distribution $\mathcal{N} C\left(0, \sigma_{n}^{2}\right)$.

The covariance matrix of data vector $\mathbf{x}(t)$ is obtained as

$$
\begin{aligned}
\mathbf{R}_{\mathbf{x x}} & =\mathrm{E}\left[\mathbf{x}(t) \mathbf{x}^{H}(t)\right]=\mathbf{A R}_{\mathbf{s s}} \mathbf{A}^{H}+\sigma_{n}^{2} \mathbf{I}_{\tilde{M}+\tilde{N}-1} \\
& =\sum_{q=1}^{Q} \sigma_{q}^{2} \mathbf{a}\left(\theta_{q}\right) \mathbf{a}^{H}\left(\theta_{q}\right)+\sigma_{n}^{2} \mathbf{I}_{\tilde{M}+\tilde{N}-1},
\end{aligned}
$$

where $\mathbf{R}_{\mathbf{s s}}=\mathrm{E}\left[\mathbf{s}(t) \mathbf{s}^{H}(t)\right]=\operatorname{diag}\left(\left[\sigma_{1}^{2}, \ldots, \sigma_{Q}^{2}\right]\right)$ is the source covariance matrix, with $\sigma_{q}^{2}$ denoting the input signal power of the $q$ th source, $q=1, \ldots, Q$. In practice, the covariance matrix is estimated using the $T$ available samples.

From antennas located at the $m$ th and $n$th positions in $\mathbf{p}$, the correlation $\mathrm{E}\left[x_{m}(t) x_{n}^{*}(t)\right]$ yields an entry in $\mathbf{R}_{\mathbf{x x}}$ with lag $p_{m}-p_{n}$. As such, all the available values of $m$ and $n$, where $0 \leq n \leq \tilde{N}-1$ and $0 \leq m \leq \tilde{M}-1$, yields virtual sensors of the following difference co-array:

$$
C_{S}=\{\mathbf{z} \mid \mathbf{z}=\mathbf{u}-\mathbf{v}, \mathbf{u} \in S, \mathbf{v} \in S\} .
$$

\section{DOA ESTIMATION}

In this section, we briefly summarize the MUSIC algorithm and CS approaches used in DOA estimation exploiting coprime arrays (refer to [2] and [4] for details).

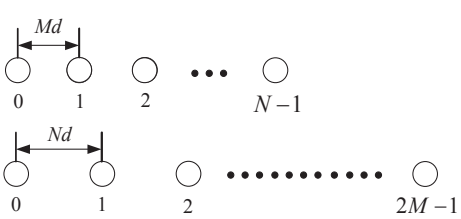

Fig. 2. The conventional coprime array configuration (Structure 1).

\subsection{MUSIC Algorithm}

Vectorizing $\mathbf{R}_{\mathbf{x x}}$ yields

$$
\mathbf{z}=\operatorname{vec}\left(\mathbf{R}_{\mathbf{x x}}\right)=\tilde{\mathbf{A}} \mathbf{b}+\sigma_{n}^{2} \tilde{\mathbf{i}}=\mathbf{B r},
$$

where $\tilde{\mathbf{A}}=\left[\tilde{\mathbf{a}}\left(\theta_{1}\right), \ldots, \tilde{\mathbf{a}}\left(\theta_{Q}\right)\right], \tilde{\mathbf{a}}\left(\theta_{q}\right)=\mathbf{a}^{*}\left(\theta_{q}\right) \otimes \mathbf{a}\left(\theta_{q}\right), \mathbf{b}=$ $\left[\sigma_{1}, \ldots, \sigma_{Q}\right]^{T}, \tilde{\mathbf{i}}=\operatorname{vec}\left(\mathbf{I}_{\tilde{M}+\tilde{N}-1}\right)$. In addition, $\mathbf{B}=[\tilde{\mathbf{A}}, \tilde{\mathbf{i}}]$ and $\mathbf{r}=\left[\mathbf{b}^{T}, \sigma_{n}^{2}\right]^{T}=\left[\sigma_{1}^{2}, \ldots, \sigma_{Q}^{2}, \sigma_{n}^{2}\right]$ are used to simplify the notations. The vector $\mathbf{z}$ amounts to the received data from a virtual array with a much longer array aperture whose corresponding steering matrix is defined by $\tilde{\mathbf{A}}$. However, the virtual source signal becomes a single snapshot of $\mathbf{b}$. Therefore, the rank of the noise-free covariance matrix of $\mathbf{z}, \mathbf{R}_{\mathbf{z z}}=\mathbf{z z}^{H}$, is one, and subspace-based DOA estimation techniques, such as MUSIC, fail to yield DOA estimates when multiple signals impinge to the array.

To overcome this problem, as shown in Fig. 2, it is proposed in [2] to double the number of array sensor in the first subarray, which has fewer sensors than the other. In this paper, we refer to this array structure as the conventional coprime array. To distinguish the notation from the prototype coprime array structure depicted in Fig. 1, we denote the number of sensors in the two subarrays as $N$ and $2 M$, respectively, where $M<N$. Because the zeroth sensor positions of the two arrays are collocated, the total number of the physical sensors in this coprime array structure is $N+2 M-1$. Note that the minimum interelement spacing of this coprime array configuration remains $\lambda / 2$. This coprime array generates all the consecutive $2 M N+2 M-1$ lag differences from $-M N-M+1$ to $M N+M-1$ [11]. By extracting these lags, we can construct a $(2 M N+2 M-1) \times(2 M N+2 M-1)$ covariance matrix, denoted as $\mathbf{R}_{\mathbf{z z}}^{\prime}$. Performing spatial smoothing yields an $(M N+M) \times(M N+M)$ full-rank covariance matrix so that the MUSIC algorithm can be used to estimate DOAs of, in theory, up to $M N+M-1$ sources.

\subsection{Compressive Sensing Approach}

Alternatively, (6) can be solved using the CS approach [4]. The desired result of $\mathbf{b}$ is represented as the solution to the following constrained $l_{1}$-norm minimization problem

$$
\hat{\mathbf{r}}^{\circ}=\arg \min _{\mathbf{r}^{\circ}}\left\|\mathbf{r}^{\circ}\right\|_{1} \quad \text { s.t. } \quad\left\|\mathbf{z}-\mathbf{B}^{\circ} \mathbf{r}^{\circ}\right\|_{2}<\epsilon,
$$

where $\epsilon$ is a user-specific bound, $\mathbf{B}^{\circ}$ is a sensing matrix consisting of the searching steering vectors and $\tilde{\mathbf{i}}$, whereas $\mathbf{r}^{\circ}$ is the sparse entries in these search grids to be determined.

This type of problems has been the objective of intensive studies in the area of CS, and a number of effective numerical 
computation methods have been developed. In this paper, we use the batch Lasso method [12], but other methods may also be used.

\section{PROPOSED COPRIME ARRAY CONFIGURATION}

We now develop CADiS configurations that maintain a large interelement spacing but have a small and consecutive equivalent spacing. Consider two uniform linear subarrays, as depicted in Fig. 3, where one consists of $N$ antennas and the other with $2 M-1$ antennas. Similar to the conventional array configuration, we assume $M$ and $N$ are coprime and $M<N$. The $N$-element subarray has an interelement spacing of $M d$, and the $(2 M-1)$-element subarray has an interelement spacing of $N d$. The difference to the conventional coprime array structure lies in the fact that these two subarrays are placed collinearly with the closest spacing between the two subarrays set to $L d$, where $L \geq M$. The total number of array sensors in the proposed coprime array structure is $2 M+N-1$, which is the same as the conventional array configuration depicted in Fig. 2. Note that the minimum interelement spacing is $M d$, as compared to $d$ in the prototype and conventional coprime arrays. In addition, the total array aperture is $(3 M N-M-2 N+L) d$, which is much larger than that of the conventional coprime array, the maximum of $(2 M-1) N d$.

In the proposed CADiS configuration, the self-differences of the two subarrays are given by the following set,

$D_{\text {self }}=\{M n d, 0 \leq n \leq N-1\} \cup\{N m d, 0 \leq m \leq 2 M-2\}$,

whereas the cross-differences between these subarrays are given by

$$
D_{\text {cross }}=\{M(N-1) d+k d+L d\},
$$

for different values of $k$ that falls into the following set,

$$
K=\{N m-M n, 0 \leq m \leq 2 M-2,0 \leq n \leq N-1\} .
$$

It is easy to confirm that $k \in[-(N-1) M, 2(M-1) N]$. The following proposition about set $K$ is useful to understand the properties of the resulting virtual sensors.

Proposition 1: Set $K$ has the following property:

(1) It contains at least $M N+M-1$ consecutive integers in the range $-(N-1) \leq k \leq M N+M-N-1$.

(2) There are holes at $-(a M+b N)$ in the negative range, where $a \geq 0, b>0$ are integers.

The proof is provided in the Appendix.

In the conventional array configuration, only non-negative lags in $D_{\text {cross }}$ are used since the negative lags form a subset of, and overlap with, the flipping of the positive counterpart. In the CADiS configuration, however, the negative lags do not generally overlap with the flipping of the positive lags because of the displacement two subarrays, necessitating the consideration of both positive and negative lags. The lags range between $L$ and $3 M N-M-2 N+L$, among which

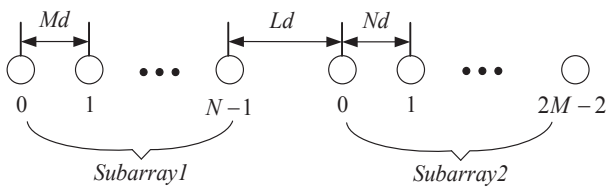

Fig. 3. Proposed CADiS configuration (Structure 2).

$M N+M-1$ are consecutive between $(M-1)(N-1)+$ $L$ and $2 M N-N-1+L$. Note that this result contains more consecutive lags than half of the result provided in [2]. On the other hand, because the coprime array has holes at $M N-M-(a M+b N)+L$, where $a \geq 0$ and $b>0$ are integers, the number of consecutive lags can be extended by choosing an approximate value of $L$ so that some selfdifference lags are aligned to the negative cross-difference holes. Specifically, a desirable choice is $L=M+N$ which results in $M N+2 M+N-1$ consecutive lags between $M N-M-N+1$ and $2 M N+M-1$. As a result, when MUSIC is used, which requires consecutive virtual array lags to perform spatial smoothing, the useable DOFs is slightly larger than a half of the conventional one.

When the CS-based approaches are used for DOA estimation, the proposed CADiS configuration provides more unique lags than the conventional one because of the utilization of negative lags. In addition, the self-differences are less likely to coincide with the cross-differences in the proposed CADiS array configuration due to the displacement between the two subarrays. Consequently, in addition to the larger virtual array aperture, it also offers a higher number of virtual sensors for improved DOA estimation. It can be shown that the number of unique lags is $3 M N+M-N$ for the conventional array, and it becomes $4 M N+2 M-1$ for the proposed array configuration when $L=M+N$ (The derivation is omitted due to space limitation).

\section{SIMULATION RESULTS}

For illustrative purposes, we compare two coprime structures i.e., the conventional array configuration (or Structure 1 for convenience) and the CADiS configuration (or Structure 2) with $L=M+N$. In both array configurations, we assume $M=6$ and $N=7$, where the unit interelement spacing is $d=$ $\lambda / 2$. Both arrays consist of 18 physical antenna sensors. The minimum spacing of the proposed array configuration is $3 \lambda$, as compared to $\lambda / 2$ in the conventional one.

The subarray sensors are respectively located at [0:6:36]d and $[0: 7: 77] d$ in Structure 1, which form a virtual array with 125 unique lags, among which 95 are consecutive. In Structure 2, the subarray sensors are located at $[0: 6: 36] d$ and [49:7:119]d. It yields a virtual array of 179 unique lags, among which 60 are consecutive. Different numbers of uncorrelated narrowband sources, which are uniformly distributed between $-50^{\circ}$ and $50^{\circ}$, are considered. We assume 512 noise-free snapshots in all simulations.

We first compare the performance of both array structures when the MUSIC technique is used. With the coprime MUSIC algorithm that exploits the consecutive virtual array lags, we can obtained 48 DOFs in Structure 1 and 30 DOFs in 
Structure 2. In Fig. 4, we consider $Q=26$ narrowband sources, which are higher than the number of physical sensors and close to the DOFs of the Structure 2. It is noted that Structure 1 resolves all 26 signals because of its high DOFs, whereas not all sources are correctly identified using Structure 2, as shown in Fig. 4. The unavailability of coprime arrays to identify all signals below its DOFs is due to the imperfect sample covariance matrix, based on which the virtual array is defined.

In the next example, the performance of MUSIC is compared to the CS based technique. For CS, higher DOFs are achieved because both consecutive and non-consecutive virtual sensors are exploited. As an example, we choose $Q=41$, which is close to the number of DOFs of Structure 1. As shown in Fig. 5, both MUSIC and CS techniques are capable to estimate all the signals in Structure 1, while for Structure 2 , only the CS technique provides successful DOA estimation since the number of the sources exceeds its DOFs usable for MUSIC. In performing the Lasso method, the regularization parameter is set to $\lambda_{t}=0.86$, and the grid interval is chosen to be $\theta_{i}^{\mathrm{g}}=0.2^{\circ}$. The results clearly show that MUSIC is less effective than CS technique when a high number of sources is present.

Finally, we compare the performance of both structures using the CS based technique. We choose $Q=51$, which exceeds the DOFs usable for MUSIC using both structures. It is evident from Fig. 6 that using Structure 2 achieved better result than Structure 1 because of the higher number of virtual elements and larger virtual aperture.

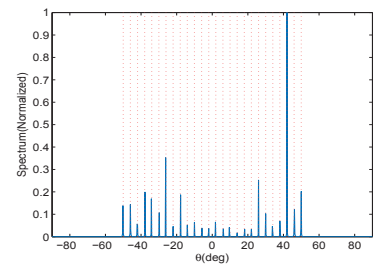

(a) Structure 1

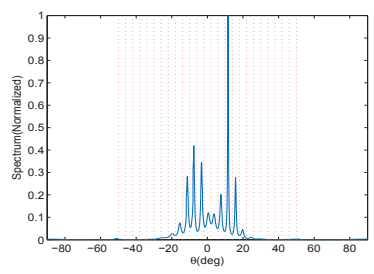

(b) Structure 2
Fig. 4. Spatial spectra estimated using coprime MUSIC for both structures $(Q=26)$.

\section{CONCLUSIONS}

We proposed effective coprime array configurations in which the minimum interelement spacing is much larger than the half-wavelength requirement. In addition to their flexibility in applications with interelement spacing constraints, the proposed configurations also achieve a higher number of degreesof-freedom to resolve more sources when compressive sensing techniques are exploited.

\section{APPENDIX}

(1) Given any integer $k$ satisfying

$$
-(N-1) \leq k \leq M N+M-N-1,
$$

we need to prove that there exist integers $m \in[0,2 M-$ $2]$ and $n \in[0, N-1]$ such that $k=m N-n M$ holds. The requirement $n \in[0, N-1]$ is equivalent to

$$
0 \leq n M \leq M N-M
$$

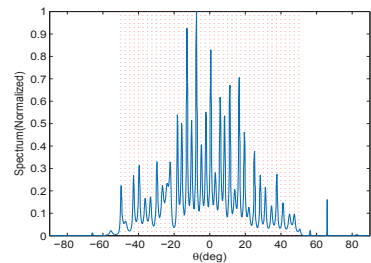

(a) MUSIC in Structure 1

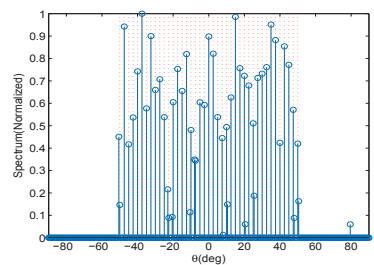

(c) Lasso in Structure 1

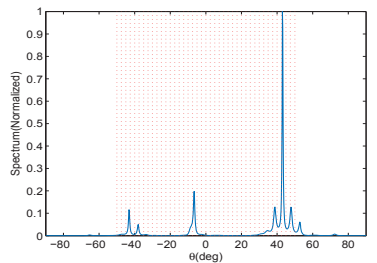

(b) MUSIC in Structure 2

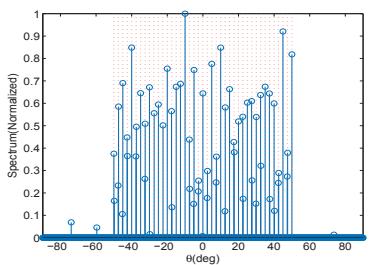

(d) Lasso in Structure 2
Fig. 5. Spatial spectra estimated using coprime MUSIC and Lasso for both structures $(Q=41)$.

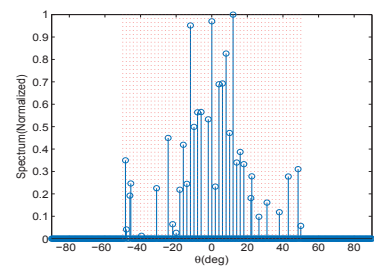

(a) Structure 1

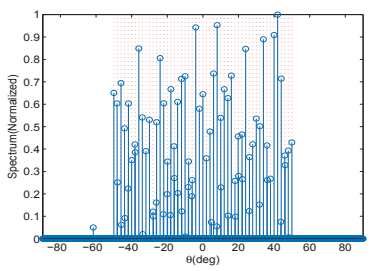

(b) Structure 2
Fig. 6. Spatial spectra estimated using the Lasso for both structures $(Q=51)$.

Because $m N=k+n M$, we obtain the following relationship by combining (11) and (12),

$$
-(N-1) \leq N m \leq 2 M N-N-1 \text {. }
$$

This result can be equivalently expressed as $-N<$ $N m<2 M N-N$, which implies $-1<m<2 M-1$. Because $m$ is an integer, this requirement is equivalent to

$$
0 \leq m \leq 2 M-2,
$$

which is satisfied in the underlying coprime array configuration.

(2) We prove the proposition by contradiction. Suppose $N m-M n=-(a M+b N)$ holds for some integers $m \in[0,2 M-2]$ and $n \in[0, N-1]$, where $a \geq 0$ and $b>0$ are integers, then relationship

$$
\frac{N}{M}=\frac{n-a}{m+b}
$$

must be valid. From $0 \leq n \leq N-1$ and $a \geq 0$, we find $n-a \leq N-1<N$. As such, due to the coprimality between $M$ and $N$, we cannot find an integer $m$ to satisfy (15). Therefore, $N m-M n \neq-(a M+b N)$, i.e., there are holes at $-(a M+b N)$ in set $K$. 


\section{REFERENCES}

[1] P. P. Vaidyanathan and P. Pal, "Sparse sensing with co-prime samplers and arrays," IEEE Trans. Signal Proc., vol. 59, no. 2, pp. 573-586, Feb. 2011.

[2] P. Pal and P. P. Vaidyanathan, "Coprime sampling and the MUSIC algorithm," in Proc. IEEE Digital Signal Proc. Workshop and IEEE Signal Proc. Education Workshop, Sedona, AZ, Jan. 2011.

[3] P. Pal and P. P. Vaidyanathan, "On application of LASSO for sparse support recovery with imperfect correlation awareness," in Proc. Asilomar Conf. Signals, Systems and Computers, Pacific Grove, CA, Nov. 2012.

[4] Y. D. Zhang, M. G. Amin, and B. Himed, "Sparsity-based DOA estimation using co-prime arrays," in Proc. IEEE ICASSP, Vancouver, Canada, May 2013.

[5] Y. D. Zhang, M. G. Amin, F. Ahmad, and B. Himed, "DOA estimation using a sparse uniform linear array with two CW signals of co-prime frequencies," in Proc. IEEE Int. Workshop on Comp. Adv. in Multi-Sensor Adaptive Proc., Saint Martin, Dec. 2013.

[6] S. Qin, Y. D. Zhang, and M. G. Amin, "DOA estimation ex- ploiting coprime frequencies," in Proc. SPIE Wireless Sensing, Localization, and Processing Conf., vol. 9109, Baltimore, MD, May 2014.

[7] A. Moffet, "Minimum-redundancy linear arrays," IEEE Trans. Antennas Propagat., vol. 16, no. 2, pp. 172-175, March 1968.

[8] W. C. Barott and P. G. Steffes, "Grating lobe reduction in aperiodic linear arrays of physically large antennas," IEEE Antennas and Wireless Propagation Letters, vol. 8, pp. 406-408, 2009.

[9] Y. Rahmat-Samii, "Reflector antennas," Chapter 15, in J. L. Volakis (Ed.), Antenna Engineering Handbook, Fourth Edition. New York, NY: McGraw-Hill, 2007.

[10] G. A. Fabrizio, High Frequency Over The Horizon Radar: Fundamental Principles, Signal Processing, and Practical Applications. New York, NY: McGraw-Hill, 2013.

[11] S. Qin, Y. D. Zhang, and M. G. Amin, "Generalized coprime array configurations," submitted to IEEE Sensor Array and Multichannel Signal Processing Workshop, A Coruña, Spain, June 2014.

[12] R. Tibshirani, "Regression shrinkage and selection via the lasso," J. Royal Statistical Society, Series B, vol. 58, no. 1, pp. 267-288, 1996. 